http://www.jfas.info

\title{
EEG SIGNAL CLASSIFICATION TO DETECT LEFT AND RIGHT COMMAND USING ARTIFICIAL NEURAL NETWORK (ANN)
}

\author{
N. Hamzah, N. A. M. Syukur, N. Zaini" and F. H. K. Zaman
}

\begin{abstract}
Faculty of Electrical Engineering, Universiti Teknologi MARA, 40450 Shah Alam, Selangor, Malaysia
\end{abstract}

Published online: 05 October 2017

\begin{abstract}
In this study, the right and left commands explored are based on the actual movement of lifting either left or right hand and the imaginary movement of lifting either left or right hand. For this initial study, EEG signals recorded based on the actual physical movements will be collected as the raw data, as well as the EEG signals recorded when imaginary movements are performed. In the scope of this research, the EEG processing focuses on analyzing two different features namely SD and ESD. These features are used as inputs to be classified by the ANN classifier. The performance of this classifier is then evaluated by measuring its accuracy in distinguishing the different interpreted commands. Based on findings from the conducted analysis, we found that PSD is the best feature to be fed as input to the ANN classifier with a high accuracy of $93 \%$ compared to when ESD feature is used as the input.
\end{abstract}

Keywords: BCI; EEG; classification; Energy Spectral Density (ESD); Power Spectral Density (PSD); Artificial Neural Network (ANN).

Author Correspondence, e-mail: drnorliza@salam.uitm.edu.my

doi: http://dx.doi.org/10.4314/jfas.v9i4s.11 


\section{INTRODUCTION}

Researchers have studied the functional brain activities that relate to the variation of surface potentials on the scalp and brain surface for many years. This signal which is called electroencephalography (EEG), has been recorded using various methods of electrode placements, filtered, amplified and processed to detect the required brain activities. The EEG signals provide rich information about the electrical activity of a human brain. EEG signal is captured from the scalp of a human, which is the electrical signal that undergoes changes in amplitude as well as in frequency while different mental tasks are performed. In this context, Brain Computer Interface (BCI) applications are useful in recognizing and classifying different mental activities. With a combination of advanced techniques of signal acquisition, preprocessing, classification and algorithm, a BCI application could be developed to assist impaired people in crying out their daily activities [1].

$\mathrm{BCI}$ is a technology that enables one to connect the brain activities of an individual to a computer. BCI allows the individual to control various devices with the right generation of brain signals without using any muscular activities. In recent years, mental tasks wave been studied by many research [2-4] and their study shows that BCI is useful especially for physically challenged individuals. Such individuals may have lost all their voluntary actions and can only rely on their cognitive actions to live their life. Having BCI systems can greatly help people especially the unprivileged ones to live their lives. The context of our study is in the scope of BCI that focuses on the interpretation of EEG signals.

The process of acquiring and processing the EEG signal from human brain is not straightforward. In this regards, artifacts and noises are removed from the raw EEG data in the pre-processing stage, after which filtering, feature extraction and normalization process are conducted. Two different features are extracted from the EEG data, specifically Energy Spectral Density (ESD) and Power Spectral Density (PSD). These features will be employed as inputs for the classification analysis. The aim of this study is to have a better understanding of the performance of Artificial Neural Network (ANN) when used as a classifier based on these two different features, i.e. specifically in classifying the left and right commands. 


\subsection{Emotiv Epoc Neuroheadset}

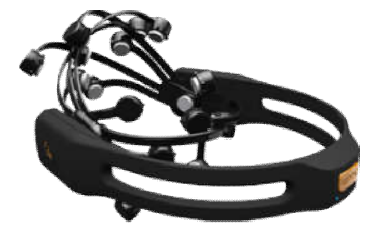

Fig.1. Emotiv Epoch Neuroheadset

Fig. 1 shows the Emotiv Epoc Neuroheadset, a model of EEG recording device that is used in this study. The headset comes with 14 independent electrodes that consist of felt pads with gold connections to increase the sensitivity of the pickups. These felt sensors need to be moist at all time to conduct the potential difference across the skull, this is done by using a saline solution [1]. Emotiv Epoc Neuroheadset is chosen as the recording device for this study due to its reliability for most application [5] and it is also low-cost.

In this scope of research, the recorded EEG signals are analyzed especially to monitor the electrical activity of sensorimotor areas of the left and right brain hemispheres. This is done by extracting a set of discriminative features to enable us to differentiate between the left and right-hand movement, for either actual or imaginary movement. The extracted features are then fed to the ANN classifier as inputs, especially to classify the left and right hand movement. Before such feature extraction process is performed, the EEG data are first filtered according to different EEG bands. Table 1 shows the different bands of EEG signal together with their frequency ranges [6-8] and description.

Table 1. Brain wave frequency range

\begin{tabular}{ccc}
\hline Band & Frequency Range (Hz) & Activity \\
\hline Delta & $0.5-4$ & Sleep \\
Theta & $4-8$ & Mental imagery \\
Alpha & $8-13$ & Relaxation, Sensory and Motor Activity \\
Beta & $13-30$ & Active Concentration and Motor Idling \\
\hline
\end{tabular}

\subsection{Imaginary Movement}

An important part of the study focuses on capturing and analyzing the recorded EEG data based on imagined movements, which are intended as commands from the brain. The activity to generate such EEG signal should not involve any physical action such as lifting the hands or moving the head. During the experiment or data collection session, the subjects are asked 
to visualize or imagine on doing a particular physical activity. To do this, the subjects are first trained and provided with proper instruction. This is to ensure that they will able to correctly perform the needed action as to allow the correct EEG signal to be recorded. This procedure involves a lot of exercises in brain activity with the subjects.

All of us know that brain is the major network control of the body functions and abilities in a human system. These mean that brain is the control unit that controls all functions or activities that human can perform such as vision, hearing and moving. This study is dedicated especially to those who have physical disabilities and having difficulties to move on their own. But despite such disabilities, they still have a normal brain function. This means that such individuals have the ability to imagine or visualize on moving their physical body. In this context, the activities in the brain generated based on a visualized physical movement have some correlation with the brain activities when the actual physical movement is done. For example, the neural signals of right and left-hand movements are controlled by the sensorimotor areas in the brain like all muscle movements in the human body. While the visualization of the hand-movement also activates the same brain areas that are normally activated when the body is preparing for and doing the real hand-movement.

\section{METHODOLOGY}

This section describes the sequence of activities carried out towards the completion of the project.

\subsection{Overall Working Principle for EEG Signal}

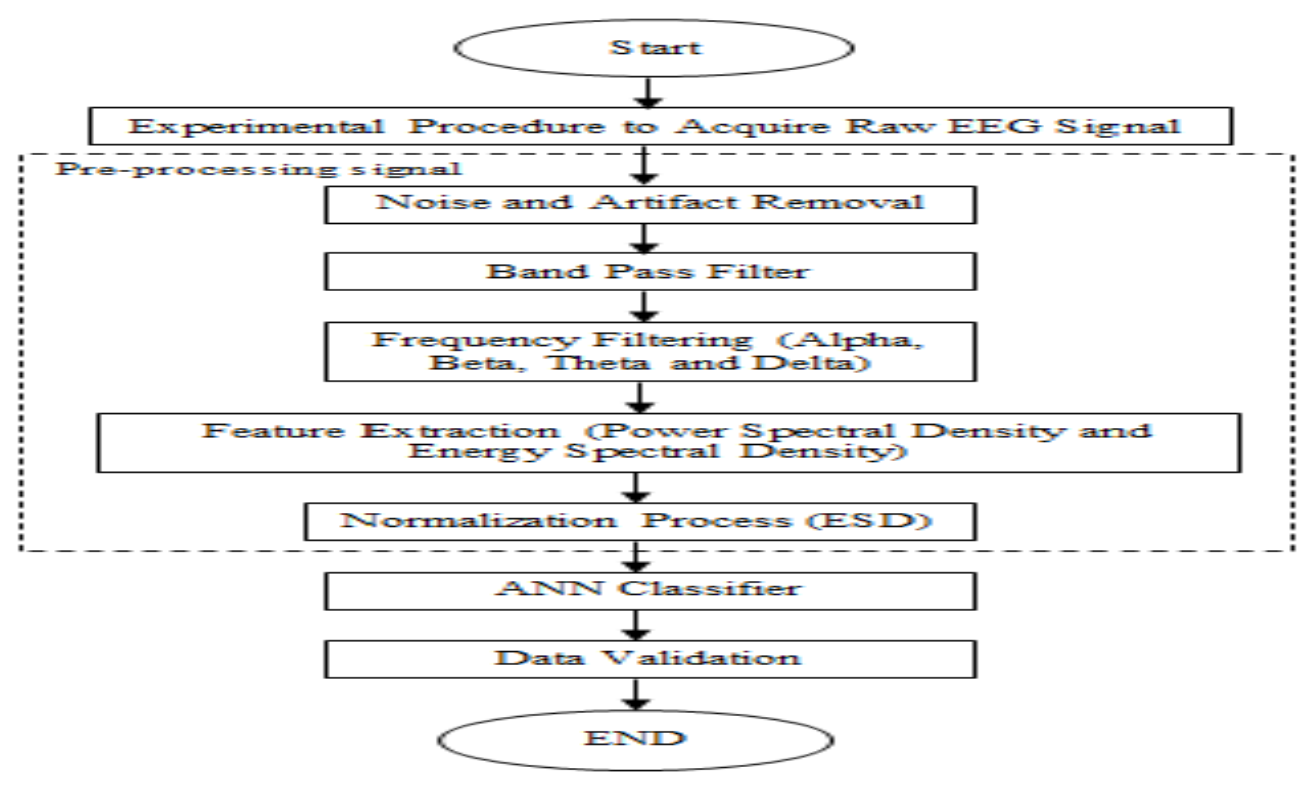

Fig.2. Activity flowchart 
Fig. 2 shows the flow of activities undergone throughout this project. The literature review activity is first conducted to gather all related information about the project. Many facts and ideas in regards to the processing and analysis of EEG signal are explored from relevant technical papers and articles. The experimental procedure is then started, where raw EEG signal data is acquired before they can be processed. For this purpose, experimental sessions are conducted with a number of subjects. For the physical-movement experiment, each subject is asked to lift their left or right hand for 30 seconds with eyes opened while for the imaginary-movement experiment the subjects are asked to imagine lifting their left or right hand with closed eyes. The detailed steps are further explained in Table 2. The activities conducted during the experimental sessions are labeled as either physical or imaginary.

\subsection{Experimental Procedure to Acquire Raw EEG Data}

Table 2. Experimental procedure

\begin{tabular}{|c|c|}
\hline Name of Activity & Type of Activity \\
\hline Physical & $\begin{array}{l}\text { Right } \\
>\text { Subjects relax their mind. } \\
\text { ( } 1 \text { minute) } \\
>\text { Subjects lift their right hand once for every } 30 \text { seconds. The data is } \\
\text { recorded and saved. This process is repeated for } 20 \text { times (open } \\
\text { eyes). } \\
>\text { Subjects relax their mind. } \\
\text { ( } 1 \text { minute) } \\
\text { Left } \\
>\text { Subjects relax their mind. } \\
\text { (1 minute) } \\
>\text { Subjects lift their left hand once for every } 30 \text { seconds. The data is } \\
\text { recorded and saved. This process is repeated for } 20 \text { times (open } \\
\text { eyes). } \\
>\text { Subjects relax their mind. } \\
\text { ( } 1 \text { minute) }\end{array}$ \\
\hline Imaginary & $\underline{\text { Right }}$ \\
\hline
\end{tabular}


Subjects relax their mind. (1 minute)

For every 30 seconds, the timer will produce a beep sound and the subject will imagine that they are lifting their right hand. The data is recorded and saved. This process is repeated for 20 times (close eyes).

Subjects relax their mind. (1 minute)

$\underline{\text { Left }}$

Subjects relax their mind. (1 minute)

For every 30 seconds, the timer will produce a beep sound and the subject will imagine that they are lifting their left hand. The data is recorded and saved. This process is repeated for 20 times (close eyes).

Subjects relax their mind. (1 minute)

\subsection{Preprocessing}

Once EEG data are recorded, the signal processing will then commenced. This first step is known as the signal pre-processing. To prepare data for this step, a special software provided by the Emotiv, i.e. the Testbench software is used in order to export the recorded EEG data to .csv files. The acquired "csv" files can then be imported to MATLAB software to be processed. In order to have a clean EEG signal, the artifacts have to be removed from the recorded data. Artifacts such as eye-blinking, electrocardiograms (ECGs), breathing from respiratory system and any other internal or external noise [9-10] will affect the result. Removal of artifacts from the EEG data is done by cutting off the signal amplitude that exceeds -100 and $100 \mu \mathrm{V}$ [11]. This is based on facts that artifacts and noises usually will generate 10 to 100 times bigger amplitude rather than the EEG data itself [11-12].

After removal of artifacts and noises, the EEG data will then be filtered into it owns frequency band by using the bandpass filter. Table 1 shows the frequency range for each band alpha, beta, theta and delta band. The bandpass filter is chosen as the filter to further group the EEG data into the different bands, with different frequency range.

\subsection{Feature Extraction}

After the signal preprocessing step, the EEG data becomes noise-free but the filtered data is still not good enough for the analysis. Thus, the feature extraction process needs to be 
performed first, especially to acquire the useful data in the form of features that are known to carry the significant information or attributes of the EEG data. Such useful data, when fed to the classifier as inputs will enable the classifier to classify the data into different groups based on the different patterns captured in the EEG signal. In this study, the features that are extracted from the EEG data are in the form of Power Spectral Density (PSD) and Energy Spectral Density (ESD) values. The captured ESD values are further normalized by using the power ratio and alpha beta ratio, while the PSD values are computed by using the mean data ratio of each band.

\subsection{Normalization}

In this study, the normalization process is done only for the extracted ESD feature. Normalization is needed to ensure that the value of the data lies in between the desired range. This technique is used to minimize the high value of power band ratio data. Equation (1) shows an example of normalization in ESD mean relative and ESD Power Ratio.

yscaled $=\frac{(\operatorname{ymax}-y \min )(x-x m i n)}{(x \operatorname{xmax}-\mathrm{xmin})}+\mathrm{ymin}$

where $\mathrm{x}$ is the target value of output data. For xmax is the maximum value of unscaled data and xmin is the minimum value of unscaled data. For ymax is the maximum value of scaled data and ymin is the minimum value of scaled data.

\subsection{ANN Classifier}

For the classification analysis, the Artificial Neural Network (ANN) is constructed and fed with either the PSD or ESD values as inputs and later evaluated for accuracy in classifying the given EEG signal data. ANN pattern classification can be categorized as either Single-layer Perceptron (SLP) or Multi-Layer Perceptron (MLP) [14]. SLP is known to have many limitations and in this regards, some researchers have found that combining the single perceptron might be useful for a larger network. Thus, for our study, MLP is chosen since it is believed to provide a better solution. The general architecture of MLP is shown in Fig. 3.

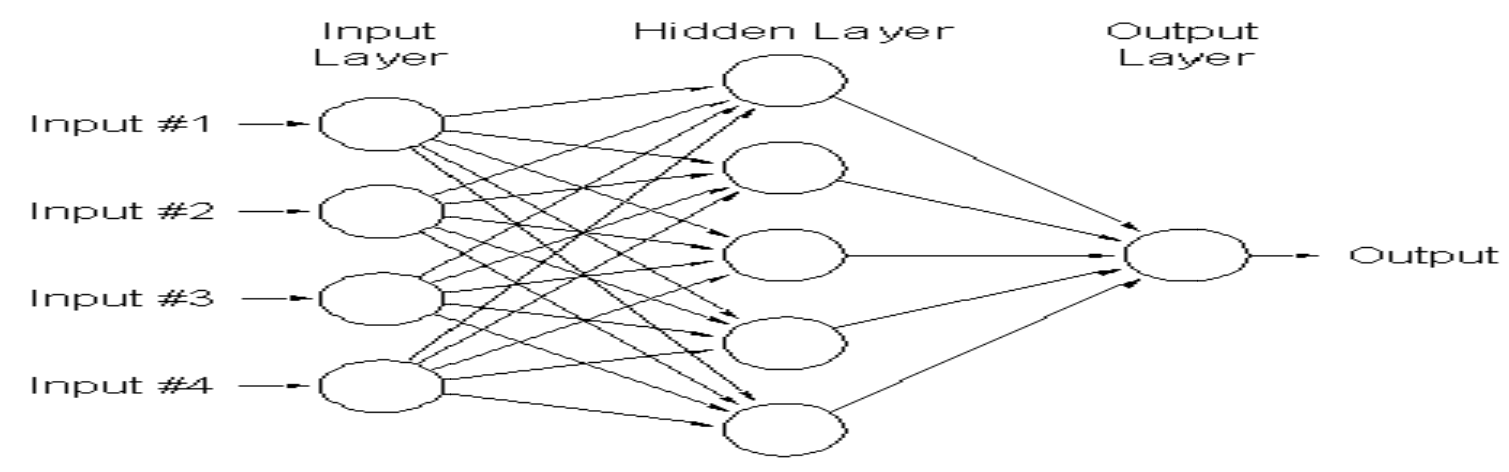

Fig.3. General architecture of MLP 
Fig. 4 shows the architecture of MLP run on the MATLAB software. This figure shows that there are 4 inputs, specifically the alpha, beta, theta and delta coefficients from feature extraction process. The number of hidden layers used in this project is set and tested between the range of 5 to 100 for all datasets. This is done especially to determine the best number of hidden units to produce the highest classification accuracy. The classification accuracy value here refers to the measure of classifier's ability to accurately classify the data for right or left-hand movement.

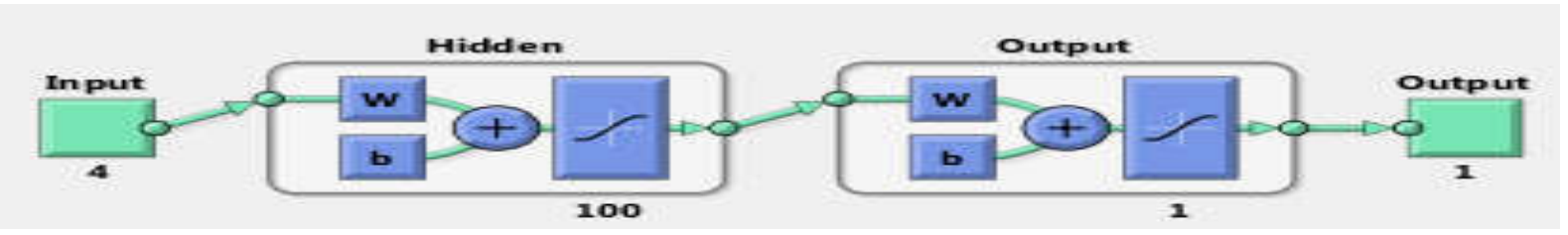

Fig.4. General architecture of MLP in MATLAB

The activation function specifically used in the ANN pattern classification is called the tan-sigmoid (Tansig) function [11]. Tansig function is usually used for hidden and output layer. Fig. 5 shows the graph of Tansig activation function where the input is between +1 and -1 range and any other value outside the range will be pushed inward within the range.

ANN pattern classification is trained by using 3 types of datasets that are physical, imaginary and combined physical and imaginary dataset. Table 3 shows the dataset representing the physical- movement. The dataset consists of data for physically lifting the left and right hand. There is a total of 40 data, in which 20 data are captured for physically lifting the left hand and another 20 for physically lifting the right hand.

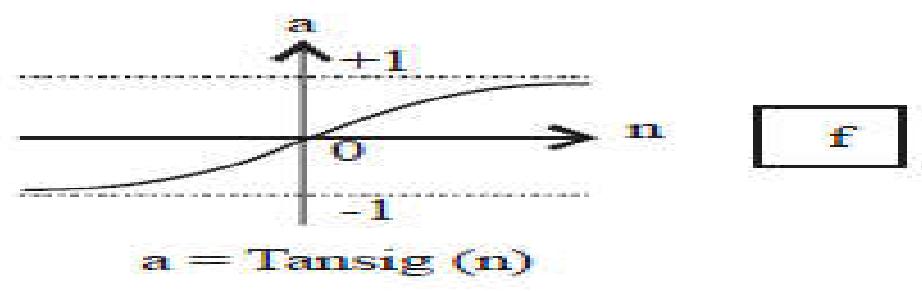

Fig.5. Tan-Sigmoid activation function

Table 3. Physical movement dataset

\begin{tabular}{cc}
\hline Type of Motor Movement & Total Data \\
\hline Left & 20 \\
Right & 20 \\
Total & 40 \\
\hline
\end{tabular}


Table 4 shows the dataset for imaginary lifting left and right hand. The total data for this set is also 40 that include 20 for imagined left-hand lifting and another 20 for imagined right-hand lifting.

Table 4. Imaginary movement dataset

\begin{tabular}{cc}
\hline Type of Motor Movement & Total Data \\
\hline Left & 20 \\
Right & 20 \\
Total & 40 \\
\hline
\end{tabular}

Table 5. Combining both physical and imaginary movement datasets

$\left.\begin{array}{cc}\hline \text { Type of Motor Movement } & \text { Total Data } \\ \hline \text { Left Physical Motor } & 20\end{array}\right\}$ Combine

Table 5 shows the dataset of combined physical and imaginary hand movement data. The combined dataset consists of 80 data where 40 data are for physically and imagined left-hand movement, while another 40 data are for physically and imagined right-hand movement. These three types of datasets will be fed as inputs to the ANN classifier. The performance of the classification process, i.e. in the form of the accuracy value will be captured and later analyzed.

The ANN pattern classification is trained by using all the three types of datasets mentioned above. By default, the dataset will be divided into training, testing and validation. The data for training, testing and validation were not manually set (following default). Because of that, the average of 10 times run is recorded between hidden unit 5 to 100 (increment of 5).

\subsection{Data Validation}

Once the EEG features are classified, the results are then validated and discussed. The important result obtained is in term of accuracy value as shown in the confusion matrix (see Fig. 6). 
The confusion matrix is used in order to illustrate and validate the results from the classification analysis. There are two output classes, which are left and right command. By referring to Fig. 6, there are 4 terms shown in the confusion matrix which are True Positive (TP), False Negative (FN), False Positive (FP) and True Negative (TN). We set the positive part as the right-hand lifting, while the negative part is set as the left-hand lifting. To elaborate, TP is the section where the network correctly predicted the right command from the data of subjects performing the right-hand movement. While $\mathrm{TN}$ is the section when the network correctly predicted the left command from the data of subjects performing the left-hand movement. FP, which is also known as "Type I error" section is when the network wrongly predicted the right command, i.e. from the data of subjects performing the left-hand movement. While FN which is also known as the "Type II error" section is when the network wrongly predicted the left command, i.e. from the data of subjects performing the right-hand movement.

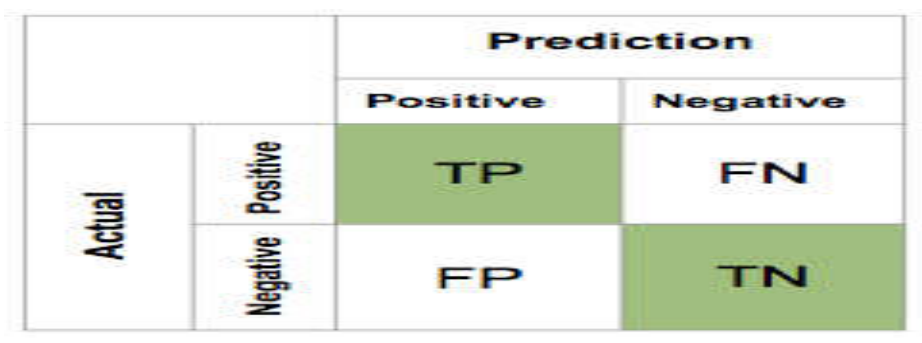

Fig.6. Confusion matrix

\section{RESULTS AND DISCUSSION}

This section presents and discusses the results obtained by the ANN classifier based on the three different datasets, i.e. i) physical-movement, ii) imaginary-movement and iii) the combination of physical and imaginary movement. Three types of EEG features are also employed as inputs for the ANN classifier specifically i) PSD, ii) ESD Mean Relative and iii) ESD Power Ratio.

Based on the different types of datasets, three different classification analysis are conducted for this study. Firstly, the classification analysis is performed based on the physical-movement dataset where the EEG data are captured based on the subjects' actual physical movement of either lifting the right or left hand. Next is the classification analysis based on the imaginary-movement dataset. The results obtained from this second analysis will be compared with the results from the first analysis on physical-movement classification. Finally, the third classification analysis is based on the combined physical and imaginary movement datasets. The main aim of conducting this analysis is to see whether there is a 
positive correlation between the physical and imaginary datasets in performing either left or right movement. The detailed results obtained for all the three classifications are shown in Table 6-8 and discussed as follow.

Table 6. Classification results based on the physical-movement dataset

\begin{tabular}{|c|c|c|c|}
\hline \multicolumn{2}{|l|}{ Hidden } & \multicolumn{2}{|c|}{ Feature as Input } \\
\hline Neurons & PSD & ESD Mean Relative & ESD Power Ratio \\
\hline 5 & $93.00 \%$ & $75.00 \%$ & $77.75 \%$ \\
\hline 10 & $88.00 \%$ & $78.00 \%$ & $79.50 \%$ \\
\hline 15 & $81.00 \%$ & $74.75 \%$ & $69.75 \%$ \\
\hline 20 & $84.00 \%$ & $82.00 \%$ & $73.00 \%$ \\
\hline 25 & $88.00 \%$ & $72.50 \%$ & $74.75 \%$ \\
\hline 30 & $88.00 \%$ & $82.50 \%$ & $77.00 \%$ \\
\hline 35 & $91.00 \%$ & $74.75 \%$ & $77.25 \%$ \\
\hline 40 & $88.00 \%$ & $75.50 \%$ & $82.50 \%$ \\
\hline 45 & $80.00 \%$ & $81.75 \%$ & $77.75 \%$ \\
\hline 50 & $90.00 \%$ & $77.25 \%$ & $80.25 \%$ \\
\hline 55 & $90.00 \%$ & $76.75 \%$ & $78.25 \%$ \\
\hline 60 & $82.00 \%$ & $69.25 \%$ & $75.75 \%$ \\
\hline 65 & $86.00 \%$ & $76.00 \%$ & $75.75 \%$ \\
\hline 70 & $92.00 \%$ & $79.50 \%$ & $74.75 \%$ \\
\hline 75 & $83.00 \%$ & $81.00 \%$ & $79.00 \%$ \\
\hline 80 & $90.00 \%$ & $76.75 \%$ & $76.00 \%$ \\
\hline 85 & $90.00 \%$ & $76.75 \%$ & $77.00 \%$ \\
\hline 90 & $85.00 \%$ & $77.75 \%$ & $72.50 \%$ \\
\hline 95 & $86.00 \%$ & $76.50 \%$ & $75.75 \%$ \\
\hline 100 & $91.00 \%$ & $72.50 \%$ & $76.50 \%$ \\
\hline
\end{tabular}

Table 6 tabulates results of the classification analysis based on the physical-movement dataset. The percentage values shown are the accuracy measures achieved by the classification analysis for each different EEG feature (as inputs) and for the different number of hidden neuron layers configured for the ANN Classifier. From the findings, it can be concluded that 
the highest accuracy value of the classification analysis is achieved with PSD data as inputs at $93 \%$, i.e. when the number of hidden layers is 5 . On the other hand, the classification analysis with ESD Mean Relative and ESD Power Ratio features as inputs achieve the highest accuracy value of $82.5 \%$ for both features, specifically for 30 and 40 hidden units respectively. From this analysis based on the physical-movement dataset, it can be concluded that PSD is the best EEG feature to be fed as input to the ANN classifier since it could produce the highest accuracy value of $93 \%$, at a low number of hidden layers.

Table 7 tabulates the results obtained from the classification analysis performed on the imaginary-movement dataset. From the findings, the highest accuracy value is obtained when PSD feature is used as the input, with the accuracy value of $76.25 \%$, i.e. for 55 hidden units. This can be compared to the classification analysis when ESD Mean Relative and ESD Power Ratio features as inputs, which obtained lower accuracy value of $64.5 \%$ and $73.75 \%$ for 30 and 70 hidden units respectively. Based on the obtained accuracy values, it is shown that the patterns encoded in the imaginary dataset are much difficult to be classified and differentiate in comparison to the physical dataset.

Table 7. Classification results based on the imaginary-movement dataset

\begin{tabular}{cccc}
\hline Hidden & \multicolumn{2}{c}{ Feature Extraction } \\
Neurons & PSD & ESD Mean Relative & ESD Power Ratio \\
\hline 5 & $68.25 \%$ & $54.50 \%$ & $56.00 \%$ \\
10 & $69.00 \%$ & $59.50 \%$ & $67.00 \%$ \\
15 & $58.25 \%$ & $56.00 \%$ & $60.50 \%$ \\
20 & $66.75 \%$ & $62.75 \%$ & $64.00 \%$ \\
25 & $71.25 \%$ & $57.50 \%$ & $69.00 \%$ \\
30 & $73.50 \%$ & $64.50 \%$ & $68.50 \%$ \\
35 & $72.00 \%$ & $56.25 \%$ & $57.75 \%$ \\
40 & $66.25 \%$ & $53.75 \%$ & $62.50 \%$ \\
45 & $70.25 \%$ & $55.50 \%$ & $63.25 \%$ \\
50 & $70.25 \%$ & $52.00 \%$ & $66.25 \%$ \\
55 & $76.25 \%$ & $56.75 \%$ & $64.75 \%$ \\
\hline
\end{tabular}




\begin{tabular}{llll}
\hline 60 & $71.75 \%$ & $56.50 \%$ & $64.50 \%$ \\
65 & $70.50 \%$ & $60.00 \%$ & $70.00 \%$ \\
70 & $62.50 \%$ & $58.25 \%$ & $73.75 \%$ \\
75 & $69.00 \%$ & $58.50 \%$ & $59.75 \%$ \\
80 & $70.00 \%$ & $57.50 \%$ & $67.00 \%$ \\
85 & $65.00 \%$ & $56.75 \%$ & $63.25 \%$ \\
90 & $74.50 \%$ & $61.00 \%$ & $60.50 \%$ \\
95 & $66.50 \%$ & $54.00 \%$ & $72.00 \%$ \\
100 & $73.75 \%$ & $55.75 \%$ & $68.00 \%$ \\
\hline
\end{tabular}

Table 8. Classification results based on the combined physical and imaginary movement dataset

\begin{tabular}{|c|c|c|c|}
\hline \multirow[t]{2}{*}{ Hidden Neurons } & \multicolumn{2}{|r|}{ Feature Extraction } & \multirow[b]{2}{*}{ ESD Power Ratio } \\
\hline & PSD & ESD Mean Relative & \\
\hline 5 & $81.01 \%$ & $63.27 \%$ & $62.77 \%$ \\
\hline 10 & $72.65 \%$ & $62.89 \%$ & $68.53 \%$ \\
\hline 15 & $84.77 \%$ & $63.90 \%$ & $69.02 \%$ \\
\hline 20 & $76.90 \%$ & $65.52 \%$ & $68.40 \%$ \\
\hline 25 & $77.15 \%$ & $65.89 \%$ & $62.66 \%$ \\
\hline 30 & $81.90 \%$ & $61.77 \%$ & $70.89 \%$ \\
\hline 35 & $74.02 \%$ & $64.40 \%$ & $71.03 \%$ \\
\hline 40 & $76.89 \%$ & $62.91 \%$ & $65.27 \%$ \\
\hline 45 & $81.27 \%$ & $65.52 \%$ & $72.78 \%$ \\
\hline 50 & $82.53 \%$ & $67.40 \%$ & $71.77 \%$ \\
\hline 55 & $74.51 \%$ & $66.01 \%$ & $63.64 \%$ \\
\hline 60 & $82.53 \%$ & $64.64 \%$ & $69.89 \%$ \\
\hline 65 & $79.42 \%$ & $64.37 \%$ & $64.76 \%$ \\
\hline 70 & $85.65 \%$ & $65.76 \%$ & $72.38 \%$ \\
\hline 75 & $80.78 \%$ & $65.15 \%$ & $74.52 \%$ \\
\hline 80 & $78.39 \%$ & $64.53 \%$ & $63.54 \%$ \\
\hline
\end{tabular}




\begin{tabular}{cccc}
\hline 85 & $79.54 \%$ & $63.03 \%$ & $69.29 \%$ \\
90 & $82.14 \%$ & $66.89 \%$ & $70.27 \%$ \\
95 & $83.78 \%$ & $62.88 \%$ & $69.91 \%$ \\
100 & $78.78 \%$ & $64.28 \%$ & $66.39 \%$ \\
\hline
\end{tabular}

Table 8 tabulates the results obtained from the classification analysis based on the combined physical and imaginary dataset. From the results, it is shown that the highest accuracy value for PSD, ESD Mean Relative and ESD Power Ratio are at $85.65 \%, 61.77 \%$ and $74.52 \%$, i.e. specifically for 70,30 and 75 hidden units respectively. It is shown that the classification analysis with ESD Mean Relative feature as input performs poorly with the low accuracy value of $62 \%$. In this analysis, once again, the PSD data leads to the best classification analysis with the highest accuracy value of $85.65 \%$. The only difference is that this high accuracy value is now obtained at a higher number of hidden unit than before (imaginary-movement dataset). By combining both physical and imaginary dataset, it is shown that the accuracy value obtained is higher when compared to the classification analysis based on only the imaginary dataset. With such findings, it is assumed that there is some degree of correlation or similarity of patterns between EEG data of physical and imaginary hand movement.

From the three different classification analysis (Table 6-8), it can be concluded that PSD work best with ANN Classifier especially when dealing with physical data set with a high accuracy value of 93\%, compared to lower accuracy measures when ESD Mean Relative and ESD Power Ratio are used as the inputs for the classification analysis.

\section{CONCLUSION}

Based on the experiment and analysis conducted in this study, PSD is found to be the best feature of EEG data to be fed as input to the ANN Classifier, compared to ESD Mean Relative and ESD Power Ratio feature values. In this context, PSD inputs have led to highest classification accuracy value for both physical and imaginary datasets and also when both physical and imaginary datasets are combined. It is also found that the number of hidden neuron layers in the classification process directly affects the accuracy measure. For instance, the classification analysis for the physical dataset with PSD values as input has obtained the 
highest accuracy value of $93 \%$ with 5 hidden layers. Whereas, for the classification analysis with ESD Power Ratio as input, the highest classification accuracy is achieved at $82.5 \%$ with 30 hidden layers. The value of hidden neuron layers employed by the ANN classifier is manually set by the programmer and the best setting can be determined following an iterative process of dynamically changing the number of hidden layers being used.

In addition, it is also found that the highest classification accuracy is achieved when the classification analysis is performed based on the physical-movement dataset. This occurs due to the fact that the amplitude of the EEG signal is found to be lower when an individual is just visualizing on doing the physical movement, compared to when they are doing the actual physical movement. A positive correlation is also found between the physical and imaginary datasets based on the classification analysis on the combined datasets. In this regards, the classification analysis based on the PSD and ESD Power Ratio features achieved accuracy value of more than $70 \%$. This indicates that there are some similarities between the patterns of brain activity caused by imaginary hand-movement with the brainwave patterns generated based on the physical hand movement. Next, further study will be aimed at studying the techniques on how to amplify the EEG [13] signals based on the imaginary command. Another target is to further explore a better technique with less computational time and high efficiency towards faster processing.

\section{ACKNOWLEDGEMENTS}

We would like to extend our acknowledgment to Universiti Teknologi MARA (UiTM) and to those who have directly and indirectly contributed to our project. This research is funded by the Fundamental Research Grants Scheme Research, Ministry of Higher Education Malaysia (FRGS/1/2014/TK03/UiTM/02/14).

\section{REFERENCES}

[1] Christopher P, Lee S, Kathryn M, Michael S. EPOC-alypse mind controlled car. Orlando: University of Central Florida, 2013

[2] Reshmi G, Amal A. Design of a BCI system for piloting a wheelchair using five class MI Based EEG. In 3rd IEEE International Conference on Advances in Computing and Communications, 2013, pp. 25-28

[3] Chai R, Ling S H, Hunter G P, Nguyen H T. Mental non-motor imagery tasks classifications of brain computer interface for wheelchair commands using genetic 
algorithm-based neural network. In IEEE International Joint Conference on Neural Networks, 2012, pp. $1-7$

[4] Sanei S, Chambers J A. Brain-Computer interfacing. In S. Sanei, \& J. A. Chambers (Eds.), EEG signal processing. England: John Wiley and Sons, 2007, pp. 239-265

[5] Amarasinghe K, Wijayasekara D, Manic M. EEG based brain activity monitoring using Artificial Neural Networks. In 7th IEEE International Conference on Human System Interactions, 2014, pp. 61-66

[6] Jahidin A H, Taib M N, Tahir N M, Ali M M, Lias S, Fuad N, Omar W R. Brainwave sub-band power ratio characteristics in intelligence assessment. In IEEE Control and System Graduate Research Colloquium, 2012, pp. 318-321

[7] Ali M M, Taib M N, Tahir N M, Jahidin A H, Yassin M. EEG sub-band spectral centroid frequencies extraction based on Hamming and equiripple filters: A comparative study. In IEEE 10th International Colloquium on Signal Processing and its Applications, 2014, pp. 199-203

[8] Jahidin A H, Taib M N, Tahir N M, Ali M M, Yassin I M, Lias S, Isa R M, Omar W R, Fuad N. Classification of intelligence quotient using EEG sub-band power ratio and ANN during mental task. In IEEE Conference on Systems, Process and Control, 2013, pp. 204-208 [9] Rao R, Derakhshani R. A comparison of EEG preprocessing methods using time delay neural networks. In 2nd International IEEE EMBS Conference on Neural Engineering, 2005, pp. 262-264

[10] Schachinger D, Schindler K, Kluge T. Automatic reduction of artifacts in EEG-signals. In 15th IEEE International Conference on Digital Signal Processing, 2007, pp. 143-146

[11] Hamzah N, Norhazman H, Zaini N, Sani M. Classification of EEG signals based on different motor movement using multi-layer perceptron artificial neural network. Journal of Biological Sciences, 2016, 16(7):265-271

[12] Soomro M H, Badruddin N, Yusoff M Z, Jatoi M A. Automatic eye-blink artifact removal method based on EMD-CCA. In IEEE International Conference on Complex Medical Engineering, 2013, pp. 186-190

[13] Ismail W O, Hanif M, Mohamed S B, Hamzah N, Rizman Z I. Human emotion detection via brain waves study by using electroencephalogram (EEG). International Journal on Advanced Science, Engineering and Information Technology, 2016, 6(6):1005-1011

[14] Yassin I M, Jailani R, Ali M, Baharom R, Hassan A, Rizman Z I. comparison between cascade forward and multi-layer perceptron neural networks for NARX functional electrical 
stimulation (FES)-based muscle model. International Journal on Advanced Science, Engineering and Information Technology, 2017, 7(1):215-221

\section{How to cite this article:}

Hamzah N, Syukur NAM, Zaini N, Zaman FHK. EEG signal classification to detect left and right command using artificial neural network (ANN). J. Fundam. Appl. Sci., 2017, 9(4S), 193-209. 